TUMOUR VIROLOGY

\section{European Conviviality}

from a Correspondent

As the old hands insisted from the outset, if there are lapses in the quality of the science presented at meetings of the European Tumour Virus Group they are more than compensated for by the pervading conviviality; and those people fortunate enough to have spent three days last week (April 19-21) at Bad Wimpfen, West Germanyvenue of the group's sixth meetingwere given good cause to continue to spread this gospel. The meeting was a considerable success in all respects, the scientific not excepted.

Dr T. Graf (Max-Planck Institute, Tübingen), for instance, reported detecting recombination between two mutant strains of Rous sarcoma virus incapable of transforming secondary chick fibroblasts. One of these mutants, of subgroup A, lacks about 15 per cent of the single stranded RNA genome of its wild type parent. The second mutant, a subgroup $\mathrm{D}$ virus carrying a point mutation, has a genome smaller than that of the subgroup A mutant. Chick fibroblasts infected by either mutant alone are not transformed but cells coinfected with both are transformed and yield progeny virus particles, some of which are recombinants. They transform chick cells, belong to subgroup D and have a genome larger than the subgroup D mutant but smaller than the subgroup A viruses.

Of the several reports from Dutch groups working on mouse mammary tumour virus (MMTV), Dr O. Mühlbock's (Netherlands Cancer Institute, Amsterdam) was outstandingly interesting. His group has found striking differences in the response to Bittner virus (C3H.MTV) and GR. MTV between congeneic strains of mice which differ by one gene only-they carry different alleles of the $\mathrm{H} 2$ histocompatibility gene. Of the mice with the $\mathrm{H} 2 \mathrm{~b}$ allele 14 per cent develop mammary tumours after being fostered on milk containing Bittner virus compared with an incidence of 89 per cent among congeneic mice carrying the $\mathrm{H} 2 \mathrm{a}$, $\mathrm{d}, \mathrm{f}, \mathrm{k}$ or $\mathrm{m}$ alleles similarly fostered. By contrast, after fostering on milks containing the GR.MTV, the incidence of mammary tumours among $\mathrm{H} 2 \mathrm{~b}$ mice is 41 per cent and among $\mathrm{H} 2 \mathrm{a}, \mathrm{d}, \mathrm{f}, \mathrm{k}$ or $\mathrm{m}$ mice 52 per cent. Clearly the antigenic composition of mice profoundly influences their response to MMTV. And Dr G. de The (International Agency for Research on Cancer, Lyon) commented that a comparable situation may exist in man. Evidence is accumulating which suggests that presence of the W5 allele of the HLA system may perhaps minimize the pathogenicity of
EB virus infections. And Dr A. Vaheri (University of Helsinki) reported experiments done in Hanafusa's laboratory (New York City), which must quicken the pulses of the manufacturers of rifampicin. At concentrations in the range $40 \mu \mathrm{g}$ to $100 \mu \mathrm{g} / \mathrm{ml}$. this antibiotic significantly inhibits the multiplication and reduces the viability of chick cells transformed by Rous sarcoma virus, whereas similar doses have no detectable effect on parallel cultures of untransformed cells.

Dr B. Hirt (Swiss Institute for Experimental Cancer Research, Lausanne) reported that polyoma virus and SV40 virus particles contain six polypeptides, three of which are also present in empty capsids. Apparently none of these polypeptides yields fingerprints identical to those of host cell histones; they may well therefore all be specified by the viral genome, in which case they would account for about 75 per cent of its total coding capacity. According to Dr J. S. Pagano and his colleagues (Swiss Institute for Experimental Cancer Research, Lausanne), one of the three polypeptides common to virions as well as empty capsids, and the three polypeptides unique to virions, may have a role in regulating transcription of the viral genome; these proteins certainly reduce the amount of RNA transcribed by Escherichia coli transcriptase.

Some adenoviruses, like polyoma virus and SV40, are oncogenic for rodents and Dr $\mathbf{J}$ Williams (Institute of Virology, Glasgow) described some of the properties of the extensive suite of adenovirus 5 temperature sensitive mutants he has isolated during the past year. They fall into nine complementation groups, and at least one mutant at its non-permissive temperature seems to lack the capacity to transform rat embryo cells, and no less than six, at the non-permissive temperature fail to induce interferon but induce it at the permissive temperature. The Glasgow group seems to have struck a rich vein.

\title{
Mammalian RNA-DNA Hybridization Improved
}

SINCE the first RNA-DNA hybridization experiment eleven years ago, the technique has been widely and successfully used in many simple organisms; however, its serious application to mammalian nucleic acids has proved to be a problem of an entirely different order of magnitude. In next Wednesday's Nature New Biology, Melli et al. describe a new method of analysis with which they have been able to demonstrate for the first time hybridization of RNA which is complementary to the non-reiterated fraction of mammalian DNA.

It is well known that the mammalian genome consists in some sequences which are highly reiterated, and others which are either unique or only repeated a few times. Previous experiments have only demonstrated hybridization of RNA with the reiterative fraction of the DNA, because hybridization with the unique fraction takes place too slowly to be observed by the usual "saturation" technique.

In any hybridization experiment in solution, RNA-DNA hybrid formation is always in competition with DNA renaturation. The membrane filter technique using immobilized DNA was introduced to overcome this problem (Gillespie and Spiegelman, J. Mol. Biol., 12, 829; 1965), but Melli and Bishop have already found (Biochem. J., 120, $225 ; 1970)$ that this method has a serious drawback in mammalian hybridization, namely that the extent of reaction is dependent on the sheared size of the DNA. They therefore reverted to the solution method, and effectively got round the problem of the two competing reactions by keeping the DNA in large excess. The theory of the method is based on the kinetic analysis of DNA renaturation devised by Britten and Kohne (Yearbook Carnegie Instn., 65, 78; 1965); the proportion of DNA renatured is a function of the parameter "Cot" (product of DNA concentration at zero time, and time), and when a segment of DNA is 50 per cent renatured the value of Cot $\left(\mathrm{Cot}_{3}\right)$ is simply the reciprocal of the DNA renaturation rate constant, the latter being a measure of genetic complexity. From this the reiteration frequency of a particular sequence can be obtained, and by keeping the DNA in excess, Melli et al. observed the RNA-DNA hybridization as a side reaction superimposed on the predominating DNA renaturation reaction.

When applied to RNA from Escherichia coli, the method worked well, but with ribosomal RNA from rat liver, agreement with the theoretical curve was not so good, suggesting that this method of hybridization is not free from inherent difficulties.

The importance of Melli et al.'s work, however, is the demonstration that rat RNA synthesized in vitro and heterogeneous nuclear RNA will hybridize with the non-reiterated fraction of rat DNA. This is something of a milestone in hybridization history, but it is a sobering comment on the complexity of mammalian nucleic acids that it has taken more than a decade of hybridization experiments for this formal demonstration to be achieved. 